\title{
Analysis of DNA relaxation and cleavage activities of recombinant Mycobacterium tuberculosis DNA topoisomerase I from a new expression and purification protocol
}

\author{
Thirunavukkarasu Annamalai, Neil Dani, Bokun Cheng and Yuk-Ching Tse- \\ Dinh*
}

Address: Department of Biochemistry and Molecular Biology, New York Medical College, Valhalla, New York, USA

Email: Thirunavukkarasu Annamalai - arasu.annamalai@gmail.com; Neil Dani - neildani@yahoo.com; Bokun Cheng - bokun_cheng@nymc.edu; Yuk-Ching Tse-Dinh* - yuk-ching_tse-dinh@nymc.edu

* Corresponding author

Published: II June 2009

BMC Biochemistry 2009, 10:18 doi:10.1 |86/147|-209/-10-18

This article is available from: http://www.biomedcentral.com//47/-209|//0//8

(C) 2009 Annamalai et al; licensee BioMed Central Ltd.

This is an Open Access article distributed under the terms of the Creative Commons Attribution License (http://creativecommons.org/licenses/by/2.0), which permits unrestricted use, distribution, and reproduction in any medium, provided the original work is properly cited.
Received: 26 March 2009

Accepted: II June 2009

\begin{abstract}
Background: Mycobacterium tuberculosis DNA topoisomerase I is an attractive target for discovery of novel TB drugs that act by enhancing the accumulation of the topoisomerase-DNA cleavage product. It shares a common transesterification domain with other type IA DNA topoisomerases. There is, however, no homology between the C-terminal DNA binding domains of Escherichia coli and M. tuberculosis DNA topoisomerase I proteins.

Results: A new protocol for expression and purification of recombinant $M$. tuberculosis DNA topoisomerase I (MtTOP) has been developed to produce enzyme of much higher specific activity than previously characterized recombinant enzyme. MtTOP was found to be less efficient than $E$. coli DNA topoisomerase I (EcTOP) in removal of remaining negative supercoils from partially relaxed DNA. DNA cleavage by MtTOP was characterized for the first time. Comparison of DNA cleavage site selectivity with EcTOP showed differences in cleavage site preferences, but the preferred sites of both enzymes have a $C$ nucleotide in the -4 position.

Conclusion: Recombinant M. tuberculosis DNA topoisomerase I can be expressed as a soluble protein and purified in high yield from $E$. coli host with a new protocol. Analysis of DNA cleavage with $M$. tuberculosis DNA substrate showed that the preferred DNA cleavage sites have a $C$ nucleotide in the -4 position.
\end{abstract}

\section{Background}

DNA topoisomerases are ubiquitous enzymes involved in the regulation of DNA supercoiling and overcoming topological barriers during replication, transcription, recombination and repair. In bacteria, the major classes of topoisomerases, type IA and type IIA, modify DNA topology by transiently cleaving and rejoining one or two strands of DNA respectively $[1,2]$. Both of these classes form a 5'-phosphotyrosyl enzyme-DNA linkage during the catalytic cycle of DNA cleavage and religation [1]. Topoisomerases are attractive targets for development of new anti-infectives [3]. Bacterial DNA gyrase and topoisomerase IV from the type IIA class are targets of antibiotics such as quinolones and fluoroquinolones. These antibiotics exhibit their bactericidal properties by trapping the covalent protein-DNA complexes formed by 
DNA gyrase or topoisomerase IV $[4,5]$. Although fluoroquinolones are effective against a broad spectrum of bacteria, alarming increase in fluoroquinolone-resistant pathogens warrants the need to develop novel drugs against new cellular targets.

Bacterial topoisomerase I, responsible for relaxing negatively supercoiled DNA, is the most common type IA topoisomerase present in almost all bacteria [6,7]. Escherichia coli topoisomerase I (EcTOP) is the well studied prototype for type IA topoisomerase [8]. EcTOP relaxes negatively supercoiled DNA through a magnesium-dependent, ATP-independent catalytic mechanism. No specific inhibitor for bacterial topoisomerase I, effective at a relevant clinical and physiological concentration, has been identified. Bacterial topoisomerase I, by virtue of its presence in nearly all bacterial genomes, and in view of its association with DNA during the vulnerable stage of cleavage-religation, could be utilized as a target for novel antimicrobials [3]. This strategy could be useful in developing drugs to treat highly fatal bacterial diseases like tuberculosis [9]. The fact that approximately one-third of the world's population is affected by tuberculosis indicates the need to develop effective drugs against this disease [10]. Also, since multiple drug resistance is common in Mycobacterium tuberculosis, it would be significant if a novel antibiotic targeting $M$. tuberculosis DNA topoisomerase I can be developed [9].

A logical first step towards finding inhibitors selective to M. tuberculosis topoisomerase I is to characterize the DNA modification ability of this enzyme. In this study, we describe a new expression and purification protocol for recombinant $M$. tuberculosis topoisomerase I capable of producing milligrams of pure protein. We also report the first detailed characterization of this enzyme with respect to its DNA cleavage sites and relaxation activity under different assay conditions.

\section{Results}

Expression and purification of M. tuberculosis topoisomerase I

Genome sequencing of $M$. tuberculosis $\mathrm{H} 37 \mathrm{Rv}$ strain has revealed the presence of topA gene Rv3646c which encodes a DNA topoisomerase I (MtTOP) comprising of 934 amino acids with an estimated molecular weight of $102.3 \mathrm{kDa}[11]$. Previously, Yang et al [12] have cloned and purified DNA topoisomerase I from M. tuberculosis Erdman strain in E. coli BL21 (DE3). Our efforts to express and purify recombinant MtTOP in E. coli BL21 (DE3) similarly by induction of the T7 promoter were frustrated by the insolubility of the expressed protein. Difficulties have also been encountered by other researchers [13] while trying to use recombinant DNA topoisomerase I gene present in genomic libraries of M. tuberculosis and Mycobacterium smegmatis to complement the temperature dependent deficiency of topoisomerase I (topA) function in E. coli strain AS17 [14]. Difference in codon usage was surmised to be one of the possible reasons behind this result [13]. We overcame these difficulties by expressing MtTOP from a recombinant plasmid pLIC-MTOP in an E. coli Arctic express (DE3)RP strain (Stratgene) at low temperatures $\left(12^{\circ} \mathrm{C}\right)$. The Arctic express (DE3)RP strain contained a chromosomally integrated T7 RNA polymerase which was expressed from the lacUV5 promoter. Induction of T7 RNA polymerase protein synthesis with IPTG resulted in the expression of the T7 promoter-driven recombinant protein. In addition, the Arctic express (DE3)RP strain expressed cold chaperonin proteins (Cpn10 and CPn60) and extra copies of tRNAs (recognizing arginine and proline codons) that facilitated the expression of recombinant proteins by overcoming issues of protein solubility and codon bias respectively.

Recombinant MtTOP was soluble and initially expressed as a hexa-histidine fusion protein only in the presence of IPTG (Figure 1). Purification of the fusion protein was achieved using nickel affinity chromatography. Subsequent SDS-PAGE analysis (Figure 2A) showed the predominant presence of only the fusion protein with the expected molecular weight. The hexa-histidine fusion tag was cleaved off by TEV protease treatment and MtTOP of high purity was eluted by increasing the potassium chloride gradient from a single-stranded DNA cellulose column (Figure 2B) [15]. The eluted fractions were pooled and dialyzed into storage buffer. Approximately 12 milligrams of purified protein was obtained from $7 \mathrm{~L}$ of bacterial culture in LB medium.

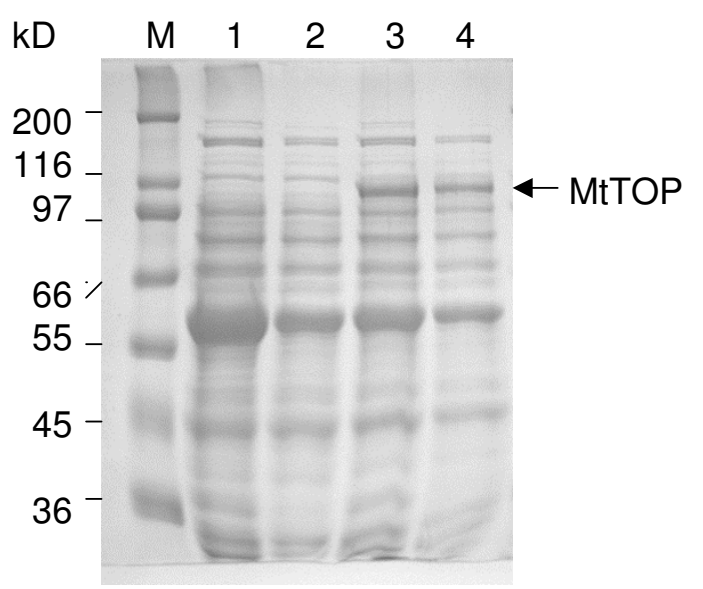

\section{Figure I}

Expression of recombinant MtTOP in E. coli Arctic express (DE3)RP strain. SDS PAGE analysis of total cell lysate (lanes I,3) and soluble cell lysate (lane 2,4) of Arctic express (DE3)RP cells transformed with PLIC-MTOP and induced with $0 \mathrm{mM}$ (lanes I,2) and I mM IPTG (lanes 3,4) at the end of 24 hours of induction in $L B$ at $12^{\circ} \mathrm{C}$. M: molecular weight standards. 


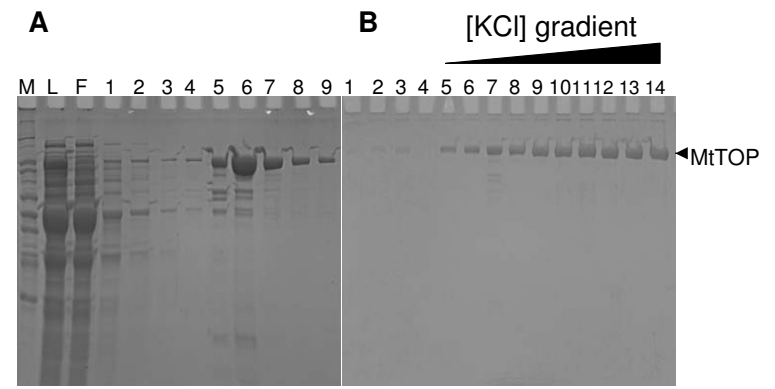

\section{Figure 2}

SDS PAGE at different stages of MtTOP purification. A. Wash (lanes I-4) and elution (lanes 5-9) fractions of fusion protein passed through a Ni-NTA agarose column. Lanes $M$ represents molecular marker and lanes $L$ and $F$ represent the initial load and flow through of the Ni-NTA agarose column before wash and elution. $\mathrm{B}$. Wash fractions (lanes I-4) and potassium chloride gradient elution fractions (lanes 5-14) of TEV digested fusion protein loaded onto single-strand DNA cellulose column.

\section{Characterization of DNA relaxation activity of $M$. tuberculosis topoisomerase I}

DNA relaxation assay was used to characterize the purified MtTOP. We compared the ability of MtTOP with that of similarly purified E.coli topoisomerase I (EcTOP) [16] in relaxing negatively supercoiled DNA by agarose gel electrophoresis. Initial assays evaluated the minimum amount of enzyme (MtTOP or ECTOP) required to bring about complete relaxation of negatively supercoiled DNA under standard conditions (Figure 3). One unit of enzyme was defined as the amount of enzyme required to relax 0.5 $\mu \mathrm{g}$ of negatively supercoiled plasmid DNA in $30 \mathrm{~min}$ at $37^{\circ} \mathrm{C}$. Results indicated that $100 \mathrm{ng}$ of EcTOP and $500 \mathrm{ng}$ of MtTOP (Figure 3A) constitute one unit of enzyme activity. However, at lower concentrations of enzyme, $=12.5$ $\mathrm{ng}$, there is no difference between the ability of MtTOP and ECTOP in removing the negative supercoils from the plasmid DNA substrate (Figure 3).

For a more detailed analysis of the relaxation activity of the purified enzymes, a time course assay with 50 ng each of MtTOP and EcTOP was performed (Figure 4). At the early time points, the rate of removal of the negative supercoils by the two enzymes was similar. However, as the plasmid DNA substrate became partially relaxed, the relaxation activity of MtTOP was less efficient than ECTOP in removing the residual negative supercoils.

It has been a well known fact that $\mathrm{Mg}^{2+}$ ions are required for the relaxation activity of bacterial type IA topoisomerases, including E. coli topoisomerase I [17,18]. We compared the $\mathrm{Mg}^{2+}$ dependence of the relaxation activity of EcTOP and MtTOP using two different enzyme concentrations (50 ng or 1 unit in a $20-\mu$ l assay) and a range of $\mathrm{Mg}^{2+}$
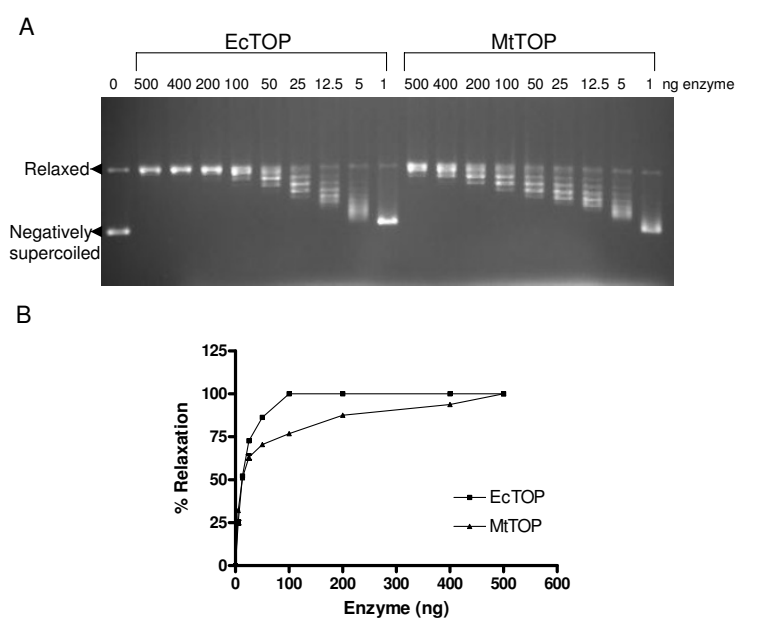

Figure 3

Relaxation of negatively supercoiled DNA to determine unit activity. A. 500 ng of negatively supercoiled plasmid DNA was subjected to relaxation as per the conditions in "materials and methods" with the indicated amount of EcTOP and MtTOP. B. DNA relaxation by different amounts of EcTOP and MtTOP was quantitated as percent relaxation for comparison. The percent relaxation was determined by dividing the distance between the negatively supercoiled band (SC); and the weighted center of the partially relaxed band (PR); by the distance between the supercoiled band (SC); and the fully relaxed band (FR). In simple terms, percent relaxation $=(\mathrm{SC}-\mathrm{PR}) /(\mathrm{SC}-\mathrm{FR})[\mathrm{I8}]$. The percent relaxation values reported are averages of at least three independent experiments. Error bars denote the standard error of mean.

levels (Figure 5). At a lower enzyme concentration (50 $\mathrm{ng})$, relaxation by EcTOP had a optimal range of $\mathrm{Mg}^{2+}$ concentrations between 2.5 to $7.5 \mathrm{mM}$ while the optimal range of $\mathrm{Mg}^{2+}$ concentrations for MtTOP was slightly higher (5-12.5 mM) (Figure 5B). Similar optimal levels of $\mathrm{Mg}^{2+}$ were found for the relaxation activities of both the EcTOP and MtTOP at higher enzyme concentrations equivalent to one unit of enzyme activity, with no relaxation observed in the absence of $\mathrm{Mg}^{2+}$ (Figure 5A). The optimal $\mathrm{Mg}^{2+}$ concentrations found here for MtTOP are higher than the $1 \mathrm{mM}$ concentration determined in previous work [12]. In other studies involving the characterization of the topoisomerase I from $M$. smegmatis, the optimal $\mathrm{Mg}^{2+}$ concentration for relaxation activity was found to be about $5 \mathrm{mM}$ [19].

\section{Mapping of DNA cleavage sites using single-stranded DNA substrates}

Although the majority of topoisomerases do not have specific sequence requirements for cleavage sites, many of them show at least a certain degree of non -randomness in cleavage site recognition $[6,20]$. For example, EcTOP and Micrococcal luteus topoisomerase I cleave the sequence 
A

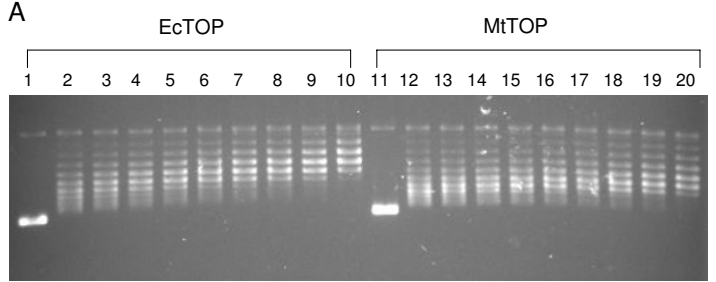

B

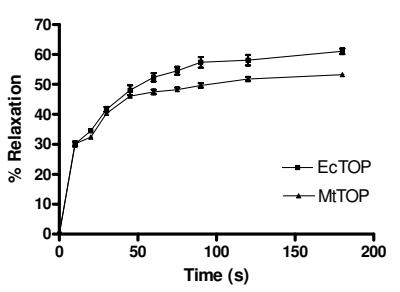

Figure 4

Time course of DNA relaxation. A. $50 \mathrm{ng}$ of EcTOP (lanes I-I0) and MtTOP (lanes II-20) was utilized in a relaxation assay as described in the "materials and methods" section over time course of $0,10,20,30,45,60,75,90,120$, $180 \mathrm{sec}$ respectively for each enzyme. B. Quantitation of the relaxation time course. The percent relaxation values reported are averages of at least three independent experiments. Error bars denote the standard error of mean.

CXXX $\downarrow$ ( $\downarrow$ represents the cleavage site) more preferentially than others [21]. Archeal and bacterial reverse gyrases, which are type IA topoisomerases, also have limited sequence requirements with only the preference of a cytosine or requirement of at least a pyrimidine at the -4 position of the cleavage site [22-24]. Previous studies elucidating the sequence specificity of topoisomerase I
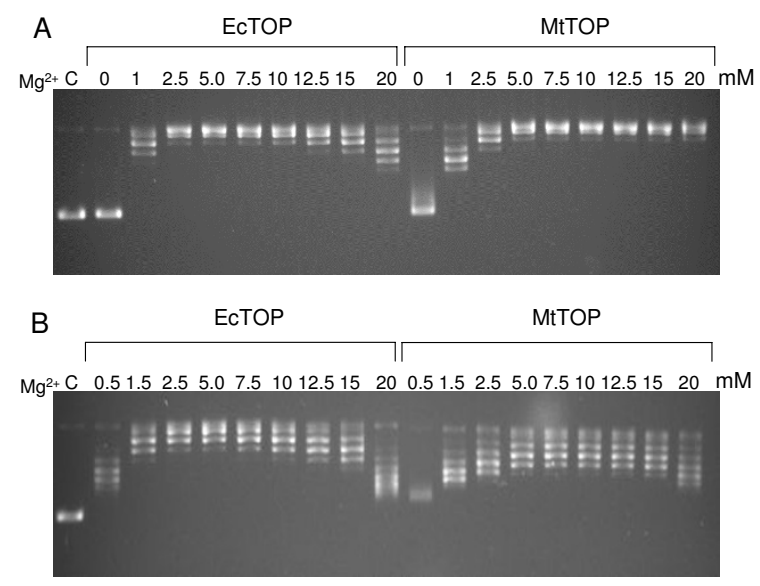

\section{Figure 5}

Effect of $\mathrm{Mg}^{2+}$ ion concentration on DNA relaxation. Effect of different concentrations of $\mathrm{Mg}^{2+}$ ion concentration ranging from 0-20 $\mathrm{mM}$ in DNA relaxation assays containing (A) high level, I enzyme unit corresponding to $100 \mathrm{ng}$ of EcTOP and $500 \mathrm{ng}$ of MtTOP; or (B) low level (50 ng) of EcTOP or MtTOP. C: control with no enzyme. from M. smegmatis reported a strong topoisomerase I site (STS), wherein the enzyme recognizes and cleaves the sequence CG/TCT $\downarrow T[25,26]$. We utilized different singlestranded 5'-32P labeled DNA substrates ranging from $\sim 200-550$ bases in length generated from either an E. coli plasmid or M. tuberculosis genomic DNA to characterize the MtTOP preferred cleavage sites. Results indicate that the DNA cleavage selectivity of MtTOP is very similar to that of EcTOP (Figure 6A, Table 1). The two enzymes share many cleavage sites on DNA derived either from $E$. coli or M. tuberculosis, but some cleavage sites were preferred by only one of these two enzymes (Figure 6B, Table $1)$. All of the cleavage sites for both enzymes were found to have a cytosine at the -4 position (CXXX $\downarrow$ ) as previously shown for many bacterial topoisomerase I enzymes $[21,27]$. There was no specific cleavage sequence recognition for MtTOP as reported for M. smegmatis topoisomerase I.

\section{Discussion}

Tuberculosis (TB) is the second leading cause of adult deaths due to infectious diseases world-wide, second only to HIV. The surge in multi-drug resistant $M$. tuberculosis makes it crucial to identify novel targets for development of new TB treatment. M. tuberculosis topoisomerase I could be one such novel target since there is only one type IA topoisomerase found in M. tuberculosis. A recent genome wide transposon mutagenesis experiment has postulated and categorised M. tuberculosis topA gene as essential [28].

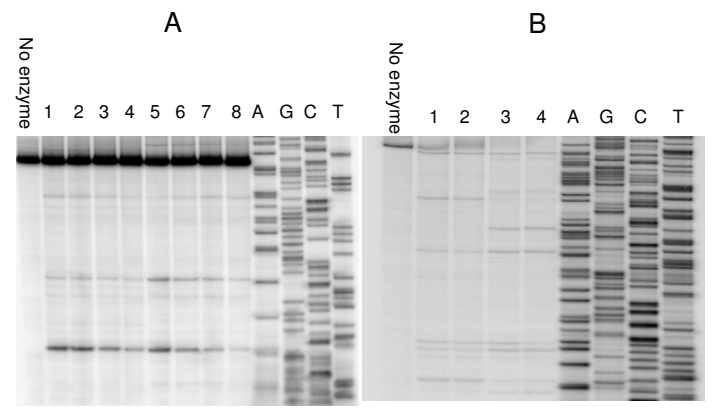

\section{Figure 6}

Mapping cleavage sites on single-stranded DNA substrate. A. Single-stranded DNA substrate Mtop (216 bases) amplified from topA gene of $M$. tuberculosis was utilized to map cleavage sites of EcTOP and MtTOP. Lanes I-4: cleavage reactions containing 400, 300, 200, $100 \mathrm{ng}$ of EcTOP respectively. Similarly lanes 5-8 contain 400, 300, 200, $100 \mathrm{ng}$ of MtTOP respectively. B. Single-stranded DNA substrate PBAD (556 bases) amplified from pBAD/thio plasmid was utilized to map cleavage sites of EcTOP and MtTOP. Lanes I-2: cleavage reactions containing 400, $300 \mathrm{ng}$ of EcTOP respectively. Similarly lanes 3-4 contain $400,300 \mathrm{ng}$ of MtTOP respectively. Lanes A, G, C, T containing the sequencing reactions with the corresponding nucleotide termination mixes were electrophoresed along with EcTOP and MtTOP cleavage reactions to determine the cleavage sites. 
Table I: List of mapped DNA cleavage sites

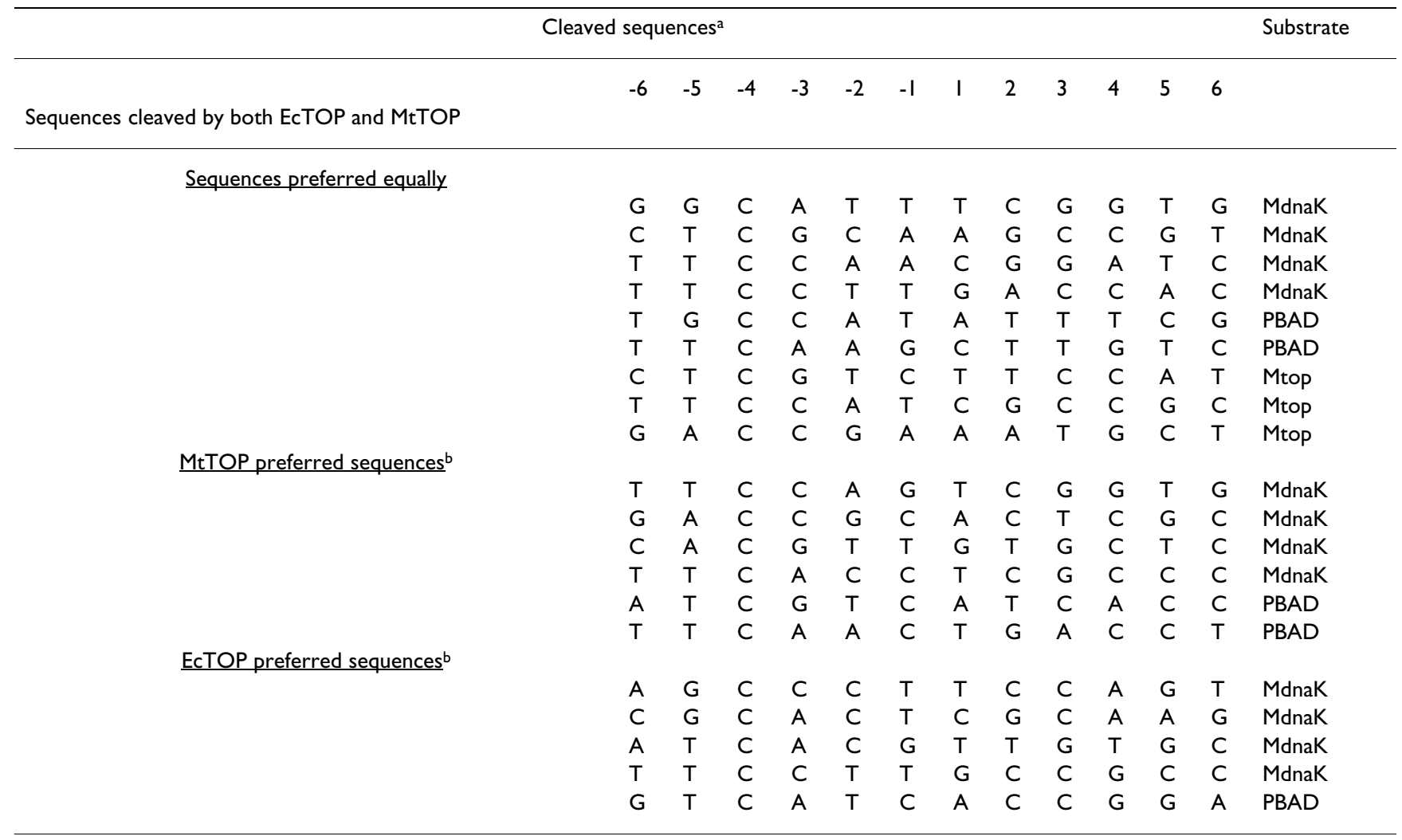

Sequences cleaved by MtTOP alone

$\begin{array}{lllllllllllll}\text { G } & \text { T } & \text { C } & \text { G } & \text { T } & \text { C } & \text { G } & \text { C } & \text { C } & \text { G } & \text { C } & \text { C } & \text { MdnaK } \\ \text { T } & \text { T } & \text { C } & \text { A } & \text { G } & \text { T } & \text { T } & \text { T } & \text { T } & \text { G } & \text { C } & \text { A } & \text { PBAD } \\ \text { G } & \text { A } & \text { C } & \text { A } & \text { G } & \text { T } & \text { G } & \text { C } & \text { A } & \text { C } & \text { C } & \text { C } & \text { PBAD }\end{array}$

Sequences cleaved by EcTOP alone

$\begin{array}{lllllllllllll}\text { A } & \text { A } & \text { C } & \text { C } & \text { T } & \text { C } & \text { A } & \text { T } & \text { C } & \text { G } & \text { G } & \text { G } & \text { MdnaK } \\ \text { C } & \text { G } & \text { C } & \text { C } & \text { G } & \text { A } & \text { G } & \text { G } & \text { T } & \text { G } & \text { G } & \text { T } & \text { MdnaK } \\ \text { C } & \text { G } & \text { C } & \text { C } & \text { A } & \text { C } & \text { T } & \text { T } & \text { C } & \text { A } & \text { C } & \text { C } & \text { PBAD }\end{array}$

a Sequences are numbered from -6 to $6\left(5^{\prime}\right.$ to $\left.3^{\prime}\right)$. Topoisomerase I mediated cleavage occurs between $-I$ and I.

b Among sequences cleaved by both EcTOP and MtTOP, sequences showing at least one-fold higher cleavage intensity towards EcTOP or MtTOP than the other enzyme are grouped as EcTOP or MtTOP preferred sequences respectively

It is also likely to be essential because every bacterium has at least one type IA topoisomerase activity. MtTOP is therefore an attractive target for drugs which would interfere with its relaxation activity (catalytic inhibitors). Moreover, besides inhibiting the overall relaxation activity of MtTOP, a more potent bactericidal effect could be achieved by drugs (catalytic poisons) that enhance the accumulation of covalent complexes on DNA, similar to the bactericidal mechanism of fluoroquinolones on type IIA bacterial topoisomerases. To aide such drug development efforts, it is important to have MtTOP protein in high purity and quantity. Here we report that by utilizing the E. coli Arctic express RP(DE3) strain, we took advan- tage of the higher GC rich codon usage efficiency and low temperature chaperone in this strain to obtain soluble MtTOP in high yield (12 mg from $7 \mathrm{~L}$ of bacterial culture). This enables future development of high through-put assays for inhibitors targeting MtTOP.

The DNA cleavage activity of MtTOP has not been characterized previously and there is a also a need for more detailed analysis of its DNA relaxation activity than in the early study of the enzyme [12]. Careful comparison with E. coli topoisomerase I (EcTOP) showed that the two enzymes had similar efficiency initially in relaxing the negatively supercoiled plasmid DNA isolated from E. coli. 
However, as the substrate plasmid DNA became partially relaxed, MtTOP was slower than EcTOP in removing the residual negative supercoils. This could be due to the different C-terminal domain found in the enzymes. The Cterminal domain found in EcTOP has been proposed to be important for substrate binding and coordination of strand passage during the relaxation cycle $[29,30]$. The Cterminal domain of MtTOP has no homology to the C-terminal domain in EcTOP, so it may function differently during the catalytic cycle. The N-terminal two-thirds, the transesterification domains of EcTOP and MtTOP have high degree of homology (41.9\% identical).

Analysis of cleavage sites on both E. coli and M. tuberculosis derived DNA substrate showed that the cleavage site preferences are quite similar with a $\mathrm{C}$ in the -4 position as have been observed for several bacterial topoisomerase I as well as archeal and bacterial reverse gyrase enzymes. It is somewhat surprising that the cleavage site preference of MtTOP is not the same as that reported for M. smegmatis topoisomerase I (CG/TCT $\downarrow T)$. It is possible that this is due to the different experimental protocols used in analysis of the cleavage sites $[25,26]$. Besides M. smegmatis topoisomerase I, there are other examples of type IA topoisomerases that have cleavage site preferences different from that of EcTOP. These include CTT $\downarrow$ for E. coli topoisomerase III [31], CANNN $\downarrow$ for human topoisomerase III [32], ANN $\downarrow$ for yeast topoisomerase III [33]. It remains unclear which part of the type IA enzyme structure determines the cleavage site selectivity. The specific sequence information for DNA cleavage by MtTOP should be useful in design of oligonucleotide substrates for DNA cleavage assays.

\section{Conclusion}

A new procedure for expression and purification of recombinant MtTOP protein in high yield has been described. The enzyme is as efficient as EcTOP in initial removal of negative supercoils from plasmid DNA, but is less efficient than EcTOP in removing the remaining negative supercoils. The preferred DNA cleavage sites of MtTOP have limited sequence specificity but contain a $\mathrm{C}$ nucleotide in the -4 position, similar to most bacterial topoisomerase I and archeal reverse gyrase cleavage sites characterized previously.

\section{Methods}

\section{MtTOP expression and purification}

MtTOP was expressed from a recombinant plasmid pLICMTOP in E.coli Arctic express (DE3)RP strain (Stratagene). MtTOP coding sequence was amplified from the genomic DNA of M. tuberculosis H37RV strain with suitable primers (LIC-Mtop5'-TACTTCCAATCCAATGCAGCTGACCCGA A AACG and LIC-Mtop3'-TTATCCACTTCCAATGTTATTAGTCGCGCTTGGCTGC) using PfuUltra II Fusion HS
DNA polymerase (Stratagene) and cloned into a vector pLIC-HK [34] through a ligation independent cloning procedure [35]. Cloning of MtTOP coding sequence into this vector containing a T7 promoter allowed T7 RNA polymerase dependent expression of MtTOP along with a tobacco etch virus (TEV) protease-cleavable $\mathrm{N}$-terminal hexahistidine tag [34]. The resulting pLIC-MTOP plasmid, capable of expressing recombinant MtTOP was first isolated in E. coli NEB Turbo competent cells (New England Biolabs) and then transformed into Arctic express (DE3)RP cells after sequence confirmation. Expression of MtTOP in transformed Arctic express (DE3)RP cells was induced by $1 \mathrm{mM}$ IPTG at $12^{\circ} \mathrm{C}$ according to the manufacturer's (Stratagene) protocol. After $24 \mathrm{~h}$ of induction, the cells were collected and subjected to freeze-thaw lysis [15] in lysis buffer $\left(50 \mathrm{mM} \mathrm{NaH}_{2} \mathrm{PO}_{4}, 300 \mathrm{mM} \mathrm{NaCl}, 10 \mathrm{mM}\right.$ imidazole, $1 \mathrm{mg} / \mathrm{ml}$ Lysozyme, $\mathrm{pH} 8.0$ ). The recombinant protein in the soluble lysate was allowed to bind to $\mathrm{Ni}$ NTA agarose (Qiagen) and packed into a column. After washing the column overnight with wash buffer $(50 \mathrm{mM}$ $\mathrm{NaH}_{2} \mathrm{PO}_{4}, 300 \mathrm{mM} \mathrm{NaCl}, 20 \mathrm{mM}$ imidazole, $\mathrm{pH} 8.0$ ), the topoisomerase protein was eluted with an elution buffer (50 mM NaH${ }_{2} \mathrm{PO}_{4}, 300 \mathrm{mM} \mathrm{NaCl}, 250 \mathrm{mM}$ Imidazole, $\mathrm{pH}$ 8.0) containing higher concentrations of imidazole. Eluted MtTOP was cleaved with TEV protease to remove the $\mathrm{N}$-terminal hexa-histidine tag and purified by passing through a single-stranded DNA cellulose column as described [15].

\section{DNA Relaxation Activity assays}

To assay for one unit of relaxation activity, EcTOP [16] and MtTOP enzymes of the same concentrations were diluted serially, ranging from 500-1 ng and assayed for DNA relaxation activity in a standard reaction volume of $20 \mu$ with $10 \mathrm{mM}$ Tris- $\mathrm{HCl}$ (pH 8.0), $50 \mathrm{mM} \mathrm{NaCl}, 0.1$ $\mathrm{mg} / \mathrm{ml}$ gelatin, $6 \mathrm{mM} \mathrm{MgCl}{ }_{2}$ and $0.5 \mu \mathrm{g}$ of supercoiled $\mathrm{pBAD} /$ thio plasmid DNA (purified by $\mathrm{CsCl}$ gradient centrifugation). After incubation at $37^{\circ} \mathrm{C}$ for $30 \mathrm{~min}$, the reactions were stopped by adding $5 \mathrm{ul}$ of $50 \mathrm{mM}$ EDTA, $50 \%$ glycerol and $0.5 \%(\mathrm{v} / \mathrm{v})$ bromophenol blue. The DNA was electrophoresed in a $1.0 \%(\mathrm{w} / \mathrm{v})$ agarose gel with TAE buffer (40 mM Tris-acetate, $\mathrm{pH} 8.1,2 \mathrm{mM}$ EDTA). The gel was stained with ethidium bromide and photographed over UV light. One unit of enzyme was defined as the least quantity of the enzyme required for complete relaxation of negatively supercoiled DNA under the given reaction conditions.

$\mathrm{Mg}^{2+}$ dependence of EcTOP and MtTOP to relax negatively supercoiled DNA was compared with either $50 \mathrm{ng}$ or one unit of enzyme (100 ng of EcTOP, $500 \mathrm{ng}$ of MtTOP) under varying concentrations of $\mathrm{MgCl}_{2}$ ranging from $0-20$ $\mathrm{mM}$ over a time period of $30 \mathrm{~min}$ at $37^{\circ} \mathrm{C}$ with similar reaction conditions as described above. Also, a low concentration (50 ng) of EcTOP and MtTOP under standard 
Table 2: Single stranded DNA substrates used

\begin{tabular}{lllcc}
\hline Substrate & Source & Primers & Substrate size (bases) & \%GC \\
\hline PBAD & pBAD/thio & Left-ATGCCATAGCATTTTTATCG & 556 \\
& Right-*GACCGGTACGCGTAGAATCG & 37 \\
MdnaK & M. tuberculosis dnaK & $\begin{array}{l}\text { Left-*GAACCCGTTGTTCTTAGACGAG } \\
\text { Might-*GGGTAACATCAAGCAGCAGAAC }\end{array}$ & $\begin{array}{l}\text { Left-*GTAGAAGTTGTTGAGCCAGT } \\
\text { Right-TACTCGTCGATCATCAAGAC }\end{array}$ & 216 \\
& M. tuberculosis topA & 62 & 61
\end{tabular}

* Primer ${ }^{32} \mathrm{P}$ labeled at 5 'end to analyze extended strand as cleavage substrate after denaturation of the PCR product. The left and right primers of MdnaK were utilized in separate experiments.

conditions ( $6 \mathrm{mM} \mathrm{MgCl}_{2}$ ) as described earlier was used to compare the ability of the respective enzymes to relax negatively supercoiled DNA at various time points of 0,10 , $20,30,45,60,75,90,120$ and $180 \mathrm{sec}$ at $37^{\circ} \mathrm{C}$.

\section{Cleavage of Single-stranded DNA}

To compare and map the cleavage sites of EcTOP and MtTOP, single stranded DNA substrates were generated first by PCR (Table 2), followed by strand denaturation. Each of these substrates were radio-labeled at the 5 ' end by having one of the corresponding forward or reverse primers labeled with $\left[\gamma_{-}{ }^{32} \mathrm{P}\right]$ ATP in the presence of T4 polynucleotide kinase prior to the PCR. The PCR products were purified using the DNA Clean and Concentrator Kit (Zymos) and eluted in TE buffer (10 mM Tris- $\mathrm{HCl}, \mathrm{pH}$ 8.0, $1 \mathrm{mM}$ EDTA). Prior to the addition of topoisomerase in the cleavage assay, the DNA substrate was denatured to single strands by heating at $95^{\circ} \mathrm{C}$ for $5 \mathrm{~min}$ and rapidly cooled on ice. After incubation with the topoisomerase at $37^{\circ} \mathrm{C}$ for $10 \mathrm{~min}$, trapping of the covalent enzyme-DNA complex and cleaved DNA was achieved by the addition of $0.1 \mathrm{M} \mathrm{NaOH}$. After neutralization, the DNA was electrophoresed in a $6 \%$ polyacrylamide sequencing gel followed by autoradiography of the dried gel to visualize the 5 '-end-labeled DNA cleavage products. DNA sequencing reaction products were generated with the same 5 ' end labeled primer corresponding to that of the substrate used in the cleavage assay and by following the cycle sequencing procedures according to the manufacturer's instructions (SequiTherm DNA sequencing Kit, Epicentre). The sequencing reaction products were electrophoresed next to lanes containing cleavage products to identify the cleavage sites.

\section{Authors' contributions}

AA carried out the relaxation and DNA cleavage assays, and drafted the manuscript. ND and BC developed and carried out the expression and purification protocol. YT conceived the study, and participated in the design and coordination and helped to draft the manuscript. All authors read and approved the final manuscript.

\section{Acknowledgements}

We wish to thank Elena Sorokin for preparation of TEV protease. This research was supported by a grant from the National Institutes of Health (ROI-GM54226) to YT.

\section{References}

I. Corbett KD, Berger JM: Structure, molecular mechanisms, and evolutionary relationships in DNA topoisomerases. Annu Rev Biophys Biomol Struct 2004, 33:95-I I8.

2. Wang JC: Cellular roles of DNA topoisomerases: a molecular perspective. Nat Rev Mol Cell Biol 2002, 3:430-440.

3. Tse-Dinh Y-: Exploring DNA Topoisomerases as Targets of Novel Therapeutic Agents in the Treatment of Infectious Diseases. Infectious Disorders - Drug Targets 2007, 7:3-9.

4. Drlica K: Mechanism of fluoroquinolone action. Curr Opin Microbiol 1999, 2:504-508.

5. Hooper DC: Bacterial topoisomerases, anti-topoisomerases, and anti-topoisomerase resistance. Clin Infect Dis 1998, 27(SuppI I):S54-63.

6. Champoux JJ: DNA topoisomerases: structure, function, and mechanism. Annu Rev Biochem 200I, 70:369-4I3.

7. Forterre P, Gribaldo S, Gadelle D, Serre M: Origin and evolution of DNA topoisomerases. Biochimie 2007, 89:427-446.

8. Tse-Dinh YC: Bacterial and archeal type I topoisomerases. Biochim Biophys Acta 1998, 1400:19-27.

9. Nagaraja V, Sikder D, Jain P: DNA topoisomerase I from mycobacteria-a potential drug target. Curr Pharm Des 2002, 8:1995-2007.

10. World Health Organization: Tuberculosis fact sheet [http:// www.who.int/mediacentre/factsheets/fs I 04/en/]

II. Cole ST, Brosch R, Parkhill J, Garnier T, Churcher C, Harris D, Gordon SV, Eiglmeier K, Gas S, Barry CE 3rd, Tekaia F, Badcock K, Basham D, Brown D, Chillingworth T, Connor R, Davies R, Devlin K, Feltwell T, Gentles S, Hamlin N, Holroyd S, Hornsby T, Jagels K, Krogh A, McLean J, Moule S, Murphy L, Oliver K, Osborne J, Quail MA, Rajandream MA, Rogers J, Rutter S, Seeger K, Skelton J, Squares R, Squares S, Sulston JE, Taylor K, Whitehead S, Barrell BG: Deciphering the biology of Mycobacterium tuberculosis from the complete genome sequence. Nature 1998, 393:537-544.

12. Yang F, Lu G, Rubin H: Cloning, expression, purification and characterization of DNA topoisomerase I of Mycobacterium tuberculosis. Gene 1996, 178:63-69.

13. Manjunatha UH, Madhusudan K, Unniraman S, Sikder D, Chatterjee $M$, Bhaduri T, Radha DR, Nagaraja V: DNA topoisomerases from Mycobacterium tuberculosis and Mycobacterium smegmatis. J Indian Inst Sci 2006, 86:75I-76I.

14. Zumstein L, Wang JC: Probing the structural domains and function in vivo of Escherichia coli DNA topoisomerase I by mutagenesis. J Mol Biol 1986, I 91 :333-340.

15. Zhu CX, Tse-Dinh YC: Overexpression and purification of bacterial DNA topoisomerase I. Methods Mol Biol I999, 94: I45-I5I.

16. Sorokin EP, Cheng B, Rathi S, Aedo SJ, Abrenica MV, Tse-Dinh YC: Inhibition of $\mathbf{M g}^{2+}$ binding and DNA religation by bacterial topoisomerase I via introduction of an additional positive charge into the active site region. Nucleic Acids Res 2008, 36:4788-4796. 
17. Wang JC: Interaction between DNA and an Escherichia coli protein omega. J Mol Biol I97I, 55:523-533.

18. Domanico PL, Tse-Dinh YC: Mechanistic studies on E. coli DNA topoisomerase I: divalent ion effects. J Inorg Biochem 199I, 42:87-96.

19. Bhaduri T, Nagaraja V: DNA topoisomerase I from Mycobacterium smegmatis. Indian J Biochem Biophys 1994, 3 I:339-343.

20. Wang JC: DNA topoisomerases. Annu Rev Biochem 1996, 65:635-692.

21. Tse YC, Kirkegaard K, Wang JC: Covalent bonds between protein and DNA. Formation of phosphotyrosine linkage between certain DNA topoisomerases and DNA. J Biol Chem 1980, 255:5560-5565.

22. Kovalsky OI, Kozyavkin SA, Slesarev Al: Archaebacterial reverse gyrase cleavage-site specificity is similar to that of eubacterial DNA topoisomerases I. Nucleic Acids Res 1990, I 8:280|-2805.

23. Jaxel $C$, Duguet $M$, Nadal $M$ : Analysis of DNA cleavage by reverse gyrase from Sulfolobus shibatae B I2. Eur J Biochem 1999, 260:103-III.

24. Bouthier de la Tour C, Amrani L, Cossard R, Neuman KC, Claude Serre M, Duguet M: Mutational analysis of the helicase-like domain of Thermotoga maritime reverse gyrase. I Biol Chem 2008, 283:27395-27402

25. Bhaduri T, Bagui TK, Sikder D, Nagaraja V: DNA topoisomerase I from Mycobacterium smegmatis. An enzyme with distinct features. J Biol Chem 1998, 273:13925-13932.

26. Sikder $D$, Nagaraja V: Determination of the recognition sequence of Mycobacterium smegmatis topoisomerase I on mycobacterial genomic sequences. Nucleic Acids Res 2000, 28: $1830-1837$

27. Viard T, Lamour V, Duguet M, Bouthier de la Tour C: Hyperthermophilic topoisomerase I from Thermotoga maritima. A very efficient enzyme that functions independently of zinc binding. J Biol Chem 200I, 276:46495-46503.

28. Sassetti CM, Rubin E): Genetic requirements for mycobacterial survival during infection. Proc Natl Acad Sci 2003, 100:12989-12994

29. Beran-Steed RK, Tse-Dinh YC: The carboxyl terminal domain of Escherichia coli DNA topoisomerase I confers higher affinity to DNA. Proteins 1989, 6:249-258.

30. Ahumada A, Tse-Dinh YC: The role of the $\mathrm{Zn}$ (II) binding domain in the mechanism of E. coli DNA topoisomerase I. BMC Biochem 2002, 3:13.

31. Zhang $H L$, DiGate $\mathrm{RJ}$ : The carboxyl-terminal residues of Escherichia coli DNA topoisomerase III are involved in substrate binding. J Biol Chem 1994, 269:9052-9059.

32. Goulaouic H, Roulon T, Flamand O, Grondard L, Lavelle F, Riou JF: Purification and characterization of human DNA topoisomerase IIlalpha. Nucleic Acids Res 1999, 27:2443-2450.

33. Kim RA, Wang JC: Identification of the yeast TOP3 gene product as a single strand-specific DNA topoisomerase. J Biol Chem 1992, 267:17178-17185.

34. Corn JE, Pease PJ, Hura GL, Berger JM: Crosstalk between primase subunits can act to regulate primer synthesis in trans. Mol Cell 2005, 20:39|-40I.

35. Doyle SA: High-throughput cloning for proteomics research. Methods Mol Biol 2005, 3 10:107-1 13.
Publish with Bio Med Central and every scientist can read your work free of charge

"BioMed Central will be the most significant development for disseminating the results of biomedical research in our lifetime. "

Sir Paul Nurse, Cancer Research UK

Your research papers will be:

- available free of charge to the entire biomedical community

- peer reviewed and published immediately upon acceptance

- cited in PubMed and archived on PubMed Central

- yours - you keep the copyright
BioMedcentral 